\title{
Communicating with HEART around the globe-does empathy transcend culture?
}

\author{
Daniella Kerbauy ${ }^{1}$, Carol Santalucia ${ }^{2}$, Rogerio Caldana ${ }^{1}$, Joe Sweet ${ }^{2}$, Jeane Tsutsui ${ }^{1}$, Edgar Rizzatti ${ }^{1}$, \\ Mary Beth Mercer $^{2}$
}

${ }^{1}$ Grupo Fleury, São Paulo, SP, Brazil; ²Cleveland Clinic, Cleveland, Ohio, USA

Contributions: (I) Conception and design: D Kerbauy, C Santalucia, J Sweet, J Tsutsui; (II) Administrative support: J Tsutsui, E Rizzatti; (III) Provision of study materials or patients: D Kerbauy, C Santalucia; (IV) Collection and assembly of data: D Kerbauy, C Santalucia; (V) Data analysis and interpretation: E Rizzatti, MB Mercer; (VI) Manuscript writing: All authors; (VII) Final approval of manuscript: All authors.

Correspondence to: Daniella Kerbauy. Grupo Fleury, Av. Gen. Valdomiro de Lima, 508 - Jabaquara, São Paulo, SP 04344-070, Brazil.

Email: daniella.kerbauy@grupofleury.com.br.

Background: Patient experience (PX) has gotten a lot of attention in the past decade and certainly plays a key role for successful healthcare delivery. Cleveland Clinic is committed to improve the PX, and has incorporated specific initiatives to create an environment that supports it. One of the most effective is Communicate with H.E.A.R.T. ${ }^{\circledR}$ which establishes a structured model for learning and demonstrating behaviors responsible for a successful service-focused culture.

Methods: Fleury Group partnered with Cleveland Clinic and adopted the Communicate with H.E.A.R.T. ${ }^{\circledR}$ program in order to improve their PX.

Results: Here, we will share findings on Fleury Group PX status and the results of skills improvement with the S.T.A.R.T. with Heart training module on the sample group of collaborators (Fleury's term for employee). Participants improved in their level of confidence on all skills trained, with a $20 \%$ overall increase in ratings of "extremely confident" after training. Then, we will review the results on skills improvement and compare and contrast the efforts in both Brazil and the United States.

Conclusions: We believe that empathy does transcend culture. This paper will address this question.

Keywords: Empathy; patient experience (PX); culture

Received: 30 August 2019; Accepted: 08 October 2019; Published: 28 November 2019.

doi: 10.21037/jhmhp.2019.10.01

View this article at: http://dx.doi.org/10.21037/jhmhp.2019.10.01

\section{Introduction}

Patient experience (PX) is defined as the sum of people's interactions during their continuum of care. PX is shaped by the service culture and how well people perceive that healthcare was delivered (1). Thus, by definition, service culture plays a key role in PX. Over the past decade, the Cleveland Clinic has made PX a top priority for the organization. The Cleveland Clinic is a nonprofit, multispecialty academic medical center that integrates clinical and hospital care with research and education. Among Cleveland Clinic's 66,000 worldwide caregivers are more than 4,200 physicians and scientists, 2,807 advanced practice providers and 16,590 registered nurses. Cleveland Clinic's health system includes a 17 -acre main campus near downtown Cleveland, as well as 18 hospitals and 210 outpatient locations throughout the world. The leadership at the Cleveland Clinic is committed to enhancing the PX and this commitment defines the Cleveland Clinic culture. Patients judge healthcare providers not only for their clinical outcomes, but also on the ability to be compassionate while delivering excellent care. As a leader in the healthcare industry, Cleveland Clinic is committed to improve the $\mathrm{PX}$, and has incorporated specific initiatives to create an environment that supports it. One of the most effective is Communicate with H.E.A.R.T. ${ }^{\circledR}$ which establishes a 
structured model for learning and demonstrating behaviors responsible for a successful service-focused culture. Cleveland Clinic noticed dramatic results by training our caregivers (Cleveland Clinic's term for employees) with easy to remember tools and skills practice.

Communicate with H.E.A.R.T. is comprised of two segments: the Foundational Program and the Sustainability Options. Although the entire Foundational Program line up consists of S.T.A.R.T. ${ }^{\circledR}$ with Heart, Respond with H.E.A.R.T. ${ }^{\circledR}$ and H.E.A.R.T. ${ }^{\circledR}$ Chats, we will concentrate on S.T.A.R.T. ${ }^{\circledR}$ with Heart as it was the focus of our collaboration with Fleury, a 92-year-old diagnostic company in Brazil.

The H.E.A.R.T. model contains service behaviors identified by patients, families, and clinical and nonclinical staff members as desirable for a great PX. These behaviors, and their associated skills, make up the content of the S.T.A.R.T. ${ }^{\circledR}$ with Heart model and are taught in the facilitator-led classes: $S$ mile and greet warmly; Tell your name, role, and what to expect; $A$ ctive listening and Assist; build Rapport and Relationships; and Thank the person. These behaviors build trust and emotional connection allowing empathy to flourish. Classes concentrate on skill building to reinforce these essential behaviors. For example, participants learn the importance of first impressions, how quickly they are made and the enormous impact nonverbal communication, such as smiling and eye contact, can have. Caregivers often feel the time they can devote to patient interaction is limited; thus, learning that non-verbal behaviors such as eye contact, physically lowering oneself or sitting to reach patient's eye level, and smiling can instantly convey empathy and compassion while providing a more positive interaction $(1,2)$. Participants also discuss the importance of a proper introduction, which includes not only their name, but also their role, and what patients can expect in the interaction (3). People are more likely to build rapport with someone when they know their name and will feel more comfortable when they can anticipate the intent of the interaction (4). The significance of active listening is another step of the model. Active listening is truly hearing another person without interrupting them, resisting the urge to jump in with thoughts or solutions, and thereby validating the experience of the patient. In addition to understanding how and why rapport and relationship building contribute to a positive PX, classes provide multiple opportunities to practice these skills and develop the ability to cultivate meaningful relationships with patients. Finally, we review what a meaningful "thank you" looks like. Skills practices are not scripted so caregivers will be more likely to incorporate them authentically into their behaviors.

The Brazilian culture is recognized as warm and welcoming. Fleury is a 92 -year-old company in the diagnostic sector in Brazil, with a long history of putting patients first. Grupo Fleury (Fleury) has been one of the most traditional medical and health organizations in the country, with diagnostic services, in different medical specialties (Clinical Pathology, Imaging, Cardiology, Endoscopy, women health, covering 37 medical specialties), that stand out for variety, complexity and quality. Fleury's operation is considered a reference by the medical community, patients and public opinion for its medical and healthcare excellence, innovation and sustainable development. Fleury's business is designed to generate value to society by promoting people's health and well-being. In addition to this vocation, Fleury has taken the responsibility of positively influencing all of its relationships, generating value and supporting the sustainability and continuity while strengthening the different resources that make up its business. Fleury participates in national and international industry and trade associations, contributing to the medical sector and to the Brazilian social and economic development. Fleury's position and purpose are expressed transparently and objectively, based on organizational values and principles of sustainability. Fleury collaborated with the Cleveland Clinic with a goal to enhance their existing culture, which was described as having "a spirit of empathy." Fleury leadership felt the skill building tools and structured framework of the Communicate with HEART program could amplify their PX. This partnership had exciting potential, but the question was asked: does empathy transcend culture? This paper will address this question. We believe it does, and we think the results of our journey together confirms it. We will examine this question in the following way. First, we will examine the PX culture at Fleury and the results of skills improvement with the S.T.A.R.T. with Heart training module on the sample group of collaborators (Fleury's term for employee). Then, we will review the results on skills improvement and compare and contrast the efforts in both Brazil and the United States

\section{Methods}

To develop a baseline of Fleury's PX journey, we utilized a four-step assessment process. The discovery process is the first step of the PX Assessment. The purpose of the 
discovery phase is to gain insight about the units from the perspective of corporate and local leadership. Using a standard list of questions, a series of telephone interviews were conducted with each of the organization's leaders. Each interview lasted approximately 30 minutes and were conducted during October and November 2017.

The following questions were used to guide the telephone interviews:

(I) Describe the culture of your organization. When we walk into your hospital, how will staff receive us?

(II) What do you see as your role in promoting PX?

(III) What is going exceptionally well in your organization related to advancing PX?

(IV) What do you see as the biggest barrier you face in delivering an excellent PX?

(V) What is the status of acceptance by the physician staff regarding the role of PX?

(VI) In thinking about our upcoming visit, what is the most important feedback you would like to receive?

Feedback from the participants identified key areas for focus:

* Opportunities with communication between departments and collaborators (Fleury's term for employees)—variation in levels of respect between clinical/non-clinical roles (possibly due to lack of technical knowledge);

* A need to present a more humanizing clinic-i.e., patient-facing areas seem very process/protocol focused (inflexible \& "robotic");

* Technical issues with Call Centers and IT-need improved software and process;

* Corporate areas of the company are not exposed to the $\mathrm{PX}$ as much;

* Are people seeking Fleury's services considered patients or clients-varying opinions;

* Lack of consistency in the PX due to growth/ acquisition—need more "Structure".

The next step was on site observation and focus groups.

\section{On-site observation}

The on-site assessment provides an opportunity for direct observation and real time discussion about PX efforts with collaborators, physicians and patients in units visited. Using a standardized assessment tool, information is gathered through observation and dialogue between the Office of Patient Experience team and Fleury's nursing staff, medical staff, ancillary staff, patients and their families. In addition to observations, two focus groups were conducted while onsite in São Paulo and Rio de Janeiro. Collaborators were chosen with help from Fleury's leadership team. The same, primarily open-ended questions were posed to all participants.

The Office of Patient Experience team conducted the observations and focus groups from December 4 through December 8, 2017. Observations lasted 60-120 minutes at most units.

And finally, as part of the assessment process, a companywide survey was distributed to all collaborators and physicians to serve as a platform for honest feedback about their work life experience within their local unit as well as the Grupo Fleury organization as a whole.

\section{Results}

\section{Fleury's PX journey}

There were 2,275 respondents to the survey; $65 \%$ spend more than $50 \%$ of their time interacting with patients. There were also several leaders at C-level and executive members who responded to the survey. It was noted that Fleury's collaborators and physicians recognized Fleury's commitment to excellence, innovation and its high-quality care in an atmosphere of patient centered service. Evidence of leadership's commitment in providing the highest quality of care for their patients was also seen throughout Cleveland Clinic's visit. Employees and physicians are passionate about and committed to their work. However, communication opportunities exist between departments and collaborators and could improve PX and employee satisfaction was observed. A need to present a more humanizing clinic, i.e., patient-facing areas seem very process/protocol focused (inflexible \& "robotic") and lack of consistency in the PX due to growth/acquisition. Concerns regarding an inordinate focus on productivity, efficiency as well as a lack of commitment to PX were also observed.

One of the ways these findings were addressed, was the Communicate with H.E.A.R.T. ${ }^{\otimes}$ program. A license agreement between Cleveland Clinic and Grupo Fleury was executed, and a group of 33 facilitators were trained in a train-the-trainer methodology in December 2017.

These facilitators started training the program to physicians and collaborators through 2018.

We conducted a survey pre- and post-training with the START with HEART program. Specifically, we examined pre-training (two weeks in advance of the course), post- 
training (one day after the course), and then again one month after the course for Cleveland Clinic and Fleury employees who registered for the Communicate with H.E.A.R.T course. Skills evaluated were: smile with heart; greet warmly; use nonverbal communication to express interest; introduce yourself; explain your role; listen with full attention; paraphrase what you heard; summarize; allow clarification; help patient or colleague; build rapport; express empathy nonverbally; give specific thanks.

\section{Fleury S.T.A.R.T. ${ }^{\circledR}$ with Heart results}

Seven hundred eighty-three Fleury collaborators (employees) were trained on the S.T.A.R.T. ${ }^{\circledR}$ with Heart program in 2018; we received 304 completed pre-training surveys and 116 post-training surveys on the S.T.A.R.T. ${ }^{\circledR}$ with Heart program.

Respondents improved in their level of confidence on all skills trained, with a $20 \%$ overall increase in ratings of "extremely confident" after training (Table 1). More than half of respondents $(170 / 304,56 \%)$ felt extremely confident on smile with heart at baseline, with an increase of $15 \%$ after training. In addition, greet warmly was one of the highest scores at baseline $(218 / 304,72 \%)$, with an increase to $82 \%$ (95/116) after training completion. Furthermore, building rapport and paraphrase skills had the highest improvement after training (32\% and $30 \%$ respectively). There was also a $22 \%$ increase in the ability to express empathy non-verbally (Table 1).

We compared these results with the data from learners in Cleveland, where we studied the results of training with participants in the Revenue Cycle Management Department who registered to S.T.A.R.T. ${ }^{\circledR}$ with Heart course between June 12, 2018 and December 14, 2018

* Pre, Post One and Post Two surveys were sent electronically with follow up for non-responders.

- Pre surveys were sent approximately two weeks in advance for each course.

- Post One surveys were sent the day after each course.

- Post Two surveys were sent one month after each course.

* Total of 967 surveys were sent for Pre, Post One and Post Two.
The following table (Table 2) shows the impact of the training on the participant's ability to convey empathy.

We were able to see an increase in confidence in the skills trained both at Cleveland Clinic and Fleury (Table 3). Although, culturally different, both Brazilian and American caregivers were positively impacted with training on S.T.A.R.T. ${ }^{\circledR}$ with Heart (see Table 3).

\section{Discussion}

Findings from our project demonstrate that employees from two different cultures felt better equipped to demonstrate empathy in their interactions with patients after completing a skills-building communication program. This is important, because empathy is foundational to making connections with our patients. Empathy-based communication helps patients to feel heard, cared for, and valued and it helps collaborators (and caregivers) relate better to each other, which ultimately benefits patients. It also helps employees reconnect with the passion that brought them to healthcare in the first place, allowing for greater engagement and fulfillment. Interestingly, aligned with Brazilian culture, we saw that smile with heart and greet warmly are behaviors more commonly expressed, with highest scores before training. In addition, training on S.T.A.R.T. ${ }^{\circledR}$ with Heart provides incremental improvement. In contrast, specific behaviors such as paraphrase and build rapport were the least confident in the Brazilian culture and the training was effective in developing these skills. Furthermore, when we matched Cleveland Clinic results to those of Fleury, skills improvement was comparable. Although specific behavior could be influenced by culture and acknowledging those differences can be very helpful to enhance empathy during the training process.

\section{Conclusions}

Findings from our project demonstrate that S.T.A.R.T. ${ }^{\circledR}$ with Heart training resulted in skills improvement on delivering an empathetic PX, regardless of cultural background. Therefore, we believe empathy transcends culture. We conclude that the skills learned in S.T.A.R.T. with Heart had a positive impact on leaners from both cultures. 
Table 1 Level of confidence in skills trained at Fleury: pre- and post-training

\begin{tabular}{|c|c|c|c|c|}
\hline Skills trained & Extremely confident (\%) & Moderately confident & Not very confident & $\%$ increase $^{\star}$ \\
\hline Smile with heart Pos & 71 & 29 & 0 & \\
\hline Greet warmly Pre & 72 & 26 & 2 & 10 \\
\hline Greet warmly Pos & 82 & 18 & 0 & \\
\hline Use nonverbal communication to express interest Pos & 60 & 38 & 2 & \\
\hline Introduce yourself Pre & 57 & 38 & 5 & 16 \\
\hline Introduce yourself Pos & 73 & 27 & 0 & \\
\hline Explain your role Pre & 64 & 34 & 2 & 17 \\
\hline Listen with full attention Pos & 63 & 36 & 1 & \\
\hline Paraphrase what you heard Pre & 27 & 69 & 4 & 30 \\
\hline Paraphrase what you heard Pos & 57 & 43 & 0 & \\
\hline Summarize Pre & 36 & 56 & 8 & 27 \\
\hline Summarize Pos & 63 & 36 & 1 & \\
\hline Allow clarification Pre & 53 & 46 & 1 & 19 \\
\hline Allow clarification Pos & 72 & 28 & 0 & \\
\hline Help patient or colleague Pre & 72 & 28 & 0 & 16 \\
\hline Give specific thanks Pre & 64 & 34 & 2 & 13 \\
\hline Give specific thanks Pos & 77 & 22 & 1 & \\
\hline Median & & & & 18 \\
\hline
\end{tabular}

*, \% increase in regards to extremely confident pre and pos training.

Table 2 Verbal and nonverbal conveyance of empathy

\begin{tabular}{lccc}
\hline & Pre & Post 1 & Post 2 \\
\hline Convey empathy verbally & $\mathrm{N}=494$ & $\mathrm{~N}=496$ & $\mathrm{~N}=277$ \\
& $53 \%$ & $73 \%$ & $\mathrm{~N} \%$ \\
Convey empathy nonverbally & $\mathrm{N}=493$ & $\mathrm{~N}=495$ & $\mathrm{~N}=274$ \\
& $47 \%$ & $70 \%$ & $65 \%$ \\
\hline
\end{tabular}


Table 3 Level of confidence on the skills trained at Fleury and Cleveland Clinic

\begin{tabular}{lcccc}
\hline \multirow{2}{*}{ Skills trained } & \multicolumn{2}{c}{ Extremely confident (\%) } & Fleury & \multicolumn{2}{c}{ Extremely confident (\%) Cleveland } \\
\cline { 2 - 5 } & Pre & Pos & Pre & 68 \\
Smile with heart & 56 & 71 & 66 & 77 \\
Greet warmly & 72 & 82 & 34 & 64 \\
Build rapport & 36 & 68 & 47 & 70 \\
Express empathy nonverbally & 44 & 66 & 60 \\
\hline
\end{tabular}

\section{Acknowledgments}

Funding: None.

\section{Footnote}

Conflicts of Interest: All authors have completed the ICMJE uniform disclosure form (available at http://dx.doi. org/10.21037/jhmhp.2019.10.01). The authors have no conflicts of interest to declare.

Ethical Statement: The authors are accountable for all aspects of the work in ensuring that questions related to the accuracy or integrity of any part of the work are appropriately investigated and resolved.

Open Access Statement: This is an Open Access article distributed in accordance with the Creative Commons Attribution-NonCommercial-NoDerivs 4.0 International License (CC BY-NC-ND 4.0), which permits the noncommercial replication and distribution of the article with the strict proviso that no changes or edits are made and the original work is properly cited (including links to both the formal publication through the relevant DOI and the license). See: https://creativecommons.org/licenses/by-nc-nd/4.0/.

\section{References}

1. Wolf AJ, Landers D, Durrah H, et al. The PX Consumer Study 2018: Insights and Opportunities for Action. Available online: https://www.theberylinstitute.org/page/ PXCONSUMERSTUDY

2. Swayden KJ, Anderson KK, Connelly LM, et al. Effect of sitting vs. standing on perception of provider time at bedside: a pilot study. Patient Educ Couns 2012;86: 166-71.

3. Boissy A, Windover AK, Bokar D, et al. Communication Skills Training for Physicians Improves Patient Satisfaction. J Gen Intern Med 2016;31:755-61.

4. Windover AK, Boissy A, Rice TW, et al. The REDE Model of Healthcare Communication: Optimizing Relationship as a Therapeutic Agent. J Patient Exp 2014;1:8-13. doi: 10.21037/jhmhp.2019.10.01

Cite this article as: Kerbauy D, Santalucia C, Caldana R, Sweet J, Tsutsui J, Rizzatti E, Mercer MB. Communicating with HEART around the globe-does empathy transcend culture? J Hosp Manag Health Policy 2019;3:30. 